\section{Authors' reply re: Peripartum hysterectomy: an economic analysis of direct healthcare costs using routinely collected data}

Sir,

Matsubara et al. ${ }^{1}$ in their Letter to the Editor raised some concerns about the conclusions and usefulness to clinicians of our paper on estimating healthcare resource use and costs associated with peripartum hysterectomy. ${ }^{2}$ The authors would like to thank them for the interest shown in the paper and for raising very important issues. We take this opportunity to provide clarifications on the specific issues they raise.

Matsubara et al. are concerned that 'although the authors cautiously refrained from stating that this extra cost is 'high', the context suggests so. This may require consideration.' We have tried to be as objective and accurate as possible in presenting and interpreting our results. Our data suggest that peripartum hysterectomy is associated with a mean total additional healthcare cost over 5 years of $£ 5380$ (£2211 if the delivery episode during which the surgery took place is excluded) ( $£$ in 2015 prices) compared with not undergoing this surgery. We are reticent about making a firm statement that this additional cost is 'high' given the absence of an external barometer of what constitutes a high additional cost in this clinical context.

On the second and third concerns raised, we would like to point out that our analysis is consistent with their suggestion that the additional costs are associated with the surgery itself or related conditions and that this may be something that might be expected. Our aim was to provide estimates of economic values for these costs, to aid in informing post-surgery service planning and delivery. For example, our data disaggregate the additional costs associated with peripartum hysterectomy across primary and secondary care encounters and across follow-up periods. However, Matsubara et al. made an important point that a comparison between alternative strategies for managing obstetric haemorrhage would have generated additional information to help choose between strategies and so be more useful to clinicians. Our data can inform such a study and future economic evaluations of the strategies listed in their letter to prevent or manage obstetric haemorrhage and related complications and so help clinicians choose cost-effective strategies for this condition.

Finally, we disagree with Matsubara et al.'s assertion that our 'conclusion was not based on the data'. Our conclusion was that 'peripartum hysterectomy is associated with increased healthcare costs during the first 5 years postpartum, primarily driven by increased inpatient hospitalisation costs in the first year postpartum. To improve outcomes for women who undergo hysterectomy and reduce healthcare service use and costs, consideration should be given to interventions that reduce avoidable repeat hospitalisations following surgery such as providing active follow up, treatment and support in the community should be considered.' The italicised section of the first sentence is based on our finding that the primary driver of healthcare costs in the hysterectomy group was increased service use during the first year postpartum (even after excluding the actual surgery itself) and the second italicised statement directly follows from that finding.

\section{References}

1 Matsubara S, Takahashi H, Matsubara D, Ohkuchi A. Re: Peripartum hysterectomy: an economic analysis of direct healthcare costs using routinely collected data. BJOG 2018; https://doi.org/10.1111/1471-0528.14103

2 Achana FA, Fleming KM, Tata LJ, Sultan AA, Petrou S. Peripartum hysterectomy: an economic analysis of direct healthcare costs using routinely collected data. BJOG 2017. https://doi.org/10.1111/1471-0528. 14950 [Epub ahead of print].

\section{Felix Achana, ${ }^{a}$ Kate Fleming,}

Laila J Tata, ${ }^{\mathrm{c}}$ Alyshah Abdul Sultan, ${ }^{\mathrm{d}}$ \& Stavros Petrou ${ }^{\mathrm{a}}$

${ }^{a}$ Clinical Trials Unit, University of Warwick, Coventry, UK ${ }^{b}$ Department of Public Health and Policy, Institute of Psychology, Health and Society, University of Liverpool, Liverpool, UK ${ }^{c}$ Faculty of Medicine and Health Sciences, University of Nottingham, Nottingham, UK ${ }^{d}$ Arthritis Research UK Primary Care Centre, Research Institute for Primary Care \& Health Sciences, Keele University, Newcastle under Lyme, UK

Accepted 13 December 2017.

DOI: 10.1111/1471-0528.15104 